LAWRENCE LIVERMORE N A T IO N A L LABORATORY

\section{Stability of a Finite-Length Screw Pinch Revisited}

D. D. Ryutov, R. H. Cohen, L. D. Pearlstein

March 24, 2004

Physics of Plasmas 
This document was prepared as an account of work sponsored by an agency of the United States Government. Neither the United States Government nor the University of California nor any of their employees, makes any warranty, express or implied, or assumes any legal liability or responsibility for the accuracy, completeness, or usefulness of any information, apparatus, product, or process disclosed, or represents that its use would not infringe privately owned rights. Reference herein to any specific commercial product, process, or service by trade name, trademark, manufacturer, or otherwise, does not necessarily constitute or imply its endorsement, recommendation, or favoring by the United States Government or the University of California. The views and opinions of authors expressed herein do not necessarily state or reflect those of the United States Government or the University of California, and shall not be used for advertising or product endorsement purposes. 


\title{
Stability of a finite-length screw pinch revisited
}

\author{
D.D. Ryutov, R.H. Cohen, L.D. Pearlstein \\ Lawrence Livermore National Laboratory, Livermore, CA 94551
}

\begin{abstract}
The ideal MHD stability of a cylindrical screw pinch (i.e., a current-carrying plasma column embedded into an external axial magnetic field) has been considered in the past in great detail. However, it turned out that there is still a gap in the analyses of this classical problem: the existing studies pertain, in fact, to an infinitely long pinch, where the axial eigenmodes can be represented as $\exp (i k z)$. The finite length is then accounted for by assigning a specific value to $k, k=2 \pi / L$, with $L$ being the distance between the electrodes; in this way, one recovers the familiar Kruskal-Shafranov (KS) stability condition. In the present paper it is emphasized that the solution of the $\exp (i k z)$ type cannot satisfy the boundary conditions at the conducting end plates. When one properly takes these boundary conditions into account, one finds a different (higher) value for the critical current. Even in the limiting case of a long-thin pinch substantial deviations from the KS condition are found. In the general case, a convenient representation is obtained for the Green's functions that express perturbations both inside and outside the plasma in terms of the radial displacement of the plasma boundary. These expressions are then used in combination with the energy principle to evaluate corrections to the long-thin approximation. The results obtained bring to a logical close one of the classical MHD problems.
\end{abstract}




\section{INTRODUCTION}

The screw pinch is a plasma column immersed into an axial magnetic field and carrying an axial current. The axial current flows between two conducting electrodes limiting the pinch along the axis. The cylindrical return conductor is situated at some distance from the surface of the plasma column (Fig. 1). We consider the magnetohydrodynamic (MHD) instability of a perfectly conducting plasma; the electrodes and the side walls are also assumed to be perfectly conducting. The fact that the axial magnetic field intersects the end surfaces does not contradict this assumption; it just means that there is a separation of time-scales: the axial magnetic field gets embedded into the end surfaces on a slow time scale, prior to plasma formation, whereas the plasma is formed and the instability develops on a short time-scale, at which the skin penetration of the current into the walls is negligible.

The stability problem in this setting is a canonical problem of ideal MHD. For an infinitely long column, one can seek a perturbation with an $\exp (i k z)$ dependence upon $z$, whereas the dependence on the azimuthal angle $\varphi$ can be sought in the form $\exp (-\operatorname{im} \varphi)$, with $m=0, \pm 1, \ldots$. For the case where current flows only along the plasma surface (perfect skin), the stability boundary is determined by a perturbation with the azimuthal mode number $m=1$, i.e., by a helical perturbation of the form expi(kz- $\varphi)$. The pitch of the most dangerous perturbation coincides with the pitch of the field line just outside the plasma, i.e, $k=B_{\varphi e} / a B_{z}$, where $B_{\varphi e}$ is the azimuthal component of the magnetic field on the plasma surface, and $B_{z}$ is the (uniform) axial magnetic field [1,2]. [This discussion corresponds to a pinch with a small axial current, so that $k a<<1$.] Further references and detailed discussions can be found in survey papers and monographs, e.g., [3,4]. 
Thus far the plasma column length was assumed to be infinite. If it is finite and equal to $L$, the usual way of incorporating $L$ into the stability analysis (e.g., [3]) is to impose periodic boundary conditions at the ends of the pinch, i.e., set

$$
k=2 \pi / L
$$

This yields the following expression for the critical current $[2,3]$ :

$$
I_{K S}=\frac{\pi a^{2} c B_{z}}{L}
$$

Here and below we are using the CGS (Gaussian) system of units. This current is usually called the "Kruskal-Shafranov current," whence the subscript "KS" in Eq. (2).

As, at $I=I_{K S}$, the pitch of the most unstable helical mode coincides with the pitch of the magnetic field line on the plasma surface, the magnetic field outside the plasma is not perturbed at all. This is why the radius of the external wall, $b$, does not enter expression (2).

Such an approach is justified in the case of a toroidal system where indeed the periodicity constraint yields a proper accounting for the finite length of the plasma. However, this approach is not quite satisfactory in the problem of a finite-length screw pinch. Namely, the boundary conditions at the conducting end plates require the plasma tangential displacement at the end plates to be zero (as, otherwise, a finite tangential electric field would have appeared at the perfectly conducting plate). On the other hand, the helical perturbation $\exp i(k z-\varphi)$ is not zero at any point along $z$, including $z=0, L$, and, accordingly, one just cannot satisfy the aforementioned boundary conditions (Fig. 2a). The assessment of this problem will be presented in this paper. We show that, indeed, proper accounting for the end boundary conditions raises the critical current above the level (2) and brings up other modifications to the standard helical solution. 
Our paper provides some new insights into the long-standing theory problem and, in this regard is of some tutorial value. It also provides tools for a quick assessment of the pinch stability in a long-thin approximation. It describes a consistent approach to stability of screw pinches with an arbitrary aspect ratio.

To put our results in context with previous studies, we note that the issue of nonperiodic boundary conditions was considered by Freidberg, Goedbloed, and Rohatgi [5], who looked into the problem of MHD stability of a toroidal plasma limited by a conducting poloidal limiter. In this case, the zero-displacement boundary conditions have to be imposed on the surface of the limiter, at some $r=r_{0}$, whereas in the inner part of the plasma one has to use a periodic boundary condition. (In Fig. 1a, this geometry would correspond to a hole of a radius $a$ in the end electrodes, with the system being periodically extended in the $z$ direction.) The authors of [5] have shown that a substantial stabilizing effect on the external kink in a "rectified" tokamak comes out of the limiter constraint. Lansky and Stchetnikov (Lansky, private communication to D. R.) have used the energy principle to consider stability of a long pinch with respect to the $m=1$ mode with rigid lateral displacements of the plasma "slices." Their stability criterion coincides with one of the results of our paper, Eq. (45). (See Ref. 6 where Lansky's and Stchetnikov result was quoted). In our analysis we use also general results of papers [4, 7], referencing them in the appropriate places of our paper.

The paper is organized as follows: In Sec. II, we present basic equations describing equilibrium and stability of the cylindrical screw pinch. We include the possibility of the presence of the hard conducting core (Fig. 1b). We assume that the axial current is a combination of a uniform current inside the pinch and a surface current on the 
plasma-vacuum interface. The next two sections, Secs. III and IV, are central for the paper. In Sec. III, a long-thin approximation, $L>>b$, is considered and a general eigenequation is derived. In Sec. IV, we provide a detailed analysis of the simplest case where there is no central insert, and where only the surface current is present. We show that, with a proper accounting for the boundary conditions, the results are quite different from those represented by Eq. (2). At the end of this section, based on the energy principle, we evaluate corrections to the long-thin approximation. In Sec. V, based on the eigenequations of Sec. III, we obtain stability criteria for various ratios of the surface-tovolume current, in the presence of the central rod. Finally, Sec. VI contains a discussion of our results. Some particularly lengthy equations are moved to the Appendix.

\section{BASIC EQUATIONS}

\section{A. Unperturbed state.}

In the unperturbed state, there is a uniformly distributed axial current inside the plasma, so that the azimuthal magnetic field is a linear function of the radius:

$$
B_{\varphi}=\frac{r}{a} B_{\varphi i}
$$

where $B_{\varphi i}$ is the azimuthal magnetic field on the inner side of the plasma boundary. If there is an axial surface current, then the magnetic field on the outer side of the plasma boundary, $B_{\varphi e}$, is different from $B_{\varphi i}$.

The relative magnitude of the uniform current inside the column with respect to the total current (which includes the surface current at the plasma-vacuum interface) can be characterized by the parameter $D$ which is the ratio of $B_{\varphi i}$ to $B_{\varphi e}$ :

$$
D=\frac{B_{\varphi i}}{B_{\varphi e}}
$$


The parameter $D$ varies in our analysis from $D=0$ (no bulk current) to $D=1$ (no surface current), Fig. 3. We do not consider a conceivable (but somewhat artificial) case of oppositely-directed bulk and surface currents $(D<0)$.

If a central conducting rod is present (Fig.1b), the current in it is assumed to be such as to maintain the dependence (3) in the plasma region; in other words, the rod current is equal to the plasma current that would have flowed in the zone occupied by the rod. The axial magnetic field is assumed to be uniform and equal to $B_{z i}$ inside the plasma and $B_{z e}$ outside the plasma. In the equilibrium,

$$
\frac{\partial p}{\partial r}+\frac{1}{2 \pi} \frac{r B_{\varphi i}^{2}}{a^{2}}=0
$$

At the boundary, in the unperturbed state,

$$
p+\frac{B_{z i}^{2}}{8 \pi}+\frac{B_{\varphi i}^{2}}{8 \pi}=\frac{B_{z e}^{2}}{8 \pi}+\frac{B_{\varphi e}^{2}}{8 \pi}
$$

The geometry of the system will be characterized by two dimensionless parameters (each less than unity):

$$
\eta=(a / b)^{2} ; \zeta=(c / a)^{2}
$$

\section{B.Perturbations}

\section{Equations in column}

The linearized equations for incompressible ideal MHD are (e.g., [3]):

$$
\begin{aligned}
& \rho \frac{\partial^{2} \xi}{\partial t^{2}}=-\nabla \delta P+\frac{1}{4 \pi}[(\mathbf{B} \cdot \nabla) \delta \mathbf{B}+(\delta \mathbf{B} \cdot \nabla) \mathbf{B}], \\
& \delta \mathbf{B}=\nabla \times \xi \times \mathbf{B}, \\
& \nabla \cdot \xi=0,
\end{aligned}
$$


where $\boldsymbol{\xi}$ is the displacement of a fluid element from its equilibrium position; other perturbations have a prefix $\delta$; the quantity $\delta P$ is defined as:

$$
\delta P=\delta p+\frac{\mathbf{B} \cdot \delta \mathbf{B}}{4 \pi} .
$$

For perturbations having the $\exp (-i \omega t-i m \varphi)$ dependence on time and azimuthal angle and for the assumed uniform current distribution inside the column [Eq. (3)], Eqs. (8) and (9) become:

$$
\begin{aligned}
& -\omega^{2} \rho \xi=-\nabla \delta P+\frac{1}{4 \pi} \hat{Q}_{i} \delta \mathbf{B}+\frac{2 B_{\varphi i}}{4 \pi a} \mathbf{e}_{z} \times \delta \mathbf{B} \\
& \delta B=\hat{Q}_{i} \xi \\
& \hat{Q}_{i} \equiv\left(B_{z i} \frac{\partial}{\partial z}-\frac{i m B_{\varphi i}}{a}\right)
\end{aligned}
$$

Note that the operator $\hat{Q}_{i}$ defined by the latter equation does not depend on $r$ and, accordingly, commutes with the differentiations over $r$. Substituting Eq. (13) into Eq. (12), one obtains:

$$
-\omega^{2} \rho \xi=-\nabla \delta P+\frac{1}{4 \pi} \hat{Q}_{i}^{2} \xi+\frac{2 B_{\varphi i} \hat{Q}_{i}}{4 \pi a} \mathbf{e}_{z} \times \xi
$$

One can express $\boldsymbol{\xi}$ in terms of $\nabla \delta P$ via a symbolic solution of Eq. (15):

$$
\begin{aligned}
& {\left[\left(\omega^{2} \rho+\frac{1}{4 \pi} \hat{Q}_{i}^{2}\right)^{2}-\left(\frac{B_{\varphi i}}{2 \pi a}\right)^{2} \hat{Q}_{i}^{2}\right] \xi=} \\
& \left(\omega^{2} \rho+\frac{1}{4 \pi} \hat{Q}_{i}^{2}\right) \nabla \delta P-\frac{B_{\varphi i} \hat{Q}_{i}}{2 \pi a} \mathbf{e}_{z} \times \nabla \delta P+\left(\frac{B_{\varphi i}}{2 \pi a}\right)^{2} \frac{\hat{Q}_{i}^{2}}{\left(\omega^{2} \rho+\frac{1}{4 \pi} \hat{Q}_{i}^{2}\right)} \mathbf{e}_{z} \frac{\partial \delta P}{\partial z}
\end{aligned}
$$

Of course, for the perturbation $\sim \exp (i k z)$, one recovers the results presented in Refs. [4] and [7]. [Note that we are seeking the helical solution of the form $\exp (i k z-i m \varphi)$.] By 
taking the divergence of this equation and using the incompressibility condition (10), one obtains a single equation for the pressure perturbation:

$$
\left(\omega^{2} \rho+\frac{1}{4 \pi} \hat{Q}_{i}^{2}\right)^{2} \nabla^{2} \delta P+\left(\frac{B_{\varphi i}}{2 \pi a}\right)^{2} \hat{Q}_{i}^{2} \frac{\partial^{2} \delta P}{\partial z^{2}}=0
$$

The boundary conditions at the end plates are those of zero plasma displacement: the normal component is zero because of the assumed impermeability of the plates; the tangential one is zero due to line-tying at the perfectly conducting walls permeated by the unperturbed normal magnetic field. Therefore,

$$
\left.\xi_{r, \varphi}\right|_{z=0, L}=0 ;\left.\quad \xi_{z}\right|_{z=0, L}=0
$$

The normal displacement must be zero also on the surface of the inner rigid cylinder (if one is present):

$$
\left.\xi_{r}\right|_{r=c}=0
$$

If there is no inner cylinder, one has to use the condition of regularity of the solution at $r=0$. At the lateral boundary of the pinch, the condition of continuity of the normal component of the momentum flux on the perturbed surface must hold. This condition is (Cf. $[3,4])$ :

$$
\delta P=\frac{\mathbf{B}_{\mathbf{e}} \cdot \delta \mathbf{B}_{e}}{4 \pi}+\frac{\xi_{r}}{4 \pi a}\left(B_{\varphi i}^{2}-B_{\varphi e}^{2}\right)
$$

\section{Vacuum region}

The magnetic field perturbation in the vacuum region is curl-free,

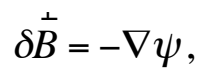

and can be determined from solving the following equation:

$$
\nabla^{2} \psi=0
$$

subject to boundary conditions (e.g., [3], [4]): 


$$
\begin{aligned}
& B_{z e} \frac{\partial \xi_{r}}{\partial z}-\frac{i m B_{\varphi e}}{a} \xi_{r}=-\frac{\partial \psi}{\partial r}, r=a \\
& \frac{\partial \psi}{\partial r}=0, r=b \\
& \frac{\partial \psi}{\partial z}=0, z=0, L
\end{aligned}
$$

corresponding to perfectly conducting material walls and the plasma.

The difficulty of the problem under consideration is related to the observation that the solution cannot be represented as the product of the functions depending only on $z$ and only on $r$. If one tries to do that, one immediately finds that it is impossible to satisfy all the boundary conditions. This inseparability of the variables makes the problem quite complex in the general case. There is, however, a limit in which the analysis becomes quite straightforward and which still yields interesting information. This is the limit of a long-thin pinch, which we consider in the next section.

\section{LONG-THIN APPROXIMATION}

In this section, we consider a pinch whose length $L$ considerably exceeds the transverse dimension, $L>>a, b$, so that there is a small parameter in the problem:

$$
\varepsilon \sim \frac{b}{L}<<1
$$

If we are no more than the order of unity beyond the stability boundary, then, as we shall see (and as it qualitatively follows from the KS criterion),

$$
\frac{B_{\varphi i, e}}{B_{z}} \sim \varepsilon
$$




\section{A.Solution in the vacuum region}

The smallness of the parameter $\varepsilon$ [Eq. (26)] allows one to neglect the term $\partial^{2} \psi / \partial z^{2}$ in the left-hand side of equation (22), because it is of order of $\varepsilon^{2}$ compared to the remaining terms. Thereby one obtains:

$$
\frac{1}{r} \frac{\partial}{\partial r} r \frac{\partial \psi}{\partial r}-\frac{m^{2}}{r^{2}} \psi=0
$$

The solution of this equation satisfying the boundary condition (24) is:

$$
\psi=C_{1}(z)\left(r^{m}+\frac{b^{2 m}}{r^{m}}\right)
$$

The $z$-dependence of the coefficient $C_{I}(z)$ is thus far undefined; what is important is that the length-scale of its variation is of order $L$, much greater than $b$. Here and below we assume that $m>0$; this does not affect results in any substantial way; it just allows us to write $m$ instead of $|m|$.

The coefficient $C_{1}$ is related to the radial displacement of the plasma boundary via the boundary condition (23):

$$
\hat{Q}_{e} \xi_{0}=\frac{m C_{1}}{a}\left(\frac{b^{2 m}}{a^{m}}-a^{m}\right),
$$

where

$$
\xi_{0}(z) \equiv \xi_{r}(r=a, z)
$$

and the operator $\hat{Q}_{e}$ is defined analogously to Eq. (14), with a substitution $i \rightarrow e$. Then, using the pressure boundary condition (20), one obtains an expression for the pressure perturbation $\delta P(a)$ in terms of the displacement of the boundary:

$$
\delta P(a)=-\frac{a}{4 \pi m} \frac{1+\eta^{m}}{1-\eta^{m}} \hat{Q}_{e}^{2} \xi_{0}+\frac{B_{\varphi i}^{2}-B_{\varphi e}^{2}}{4 \pi a} \xi_{0}
$$




\section{B.Solution inside the plasma}

By inspecting the relative value of the terms in Eq. (17), one finds that the last term contains a small parameter $\varepsilon^{2}$. We, therefore, neglect it. Likewise, one can neglect the second derivative with respect to $z$ in $\nabla^{2}$. The remaining equation,

$$
\left(\omega^{2} \rho+\frac{1}{4 \pi} \hat{Q}_{i}^{2}\right)^{2} \nabla_{\perp}^{2} \delta P=0
$$

contains two operators acting on $\delta P,\left(\omega^{2} \rho+\frac{1}{4 \pi} \hat{Q}_{i}^{2}\right)^{2}$ and $\nabla_{\perp}^{2}$. The eigenfunction of the outer one corresponds to Alfvén modes propagating independently over the various flux surfaces (this operator does not depend on $r$, so that its eigenfunction can be an arbitrary function of $r$ ). One can check that the $z$-dependence of the unstable solution that we eventually find does not make this operator zero. So, we conclude that Eq. (33) reduces to

$$
\nabla_{\perp}^{2} \delta P \equiv \frac{1}{r} \frac{\partial}{\partial r} r \frac{\partial \delta P}{\partial r}-\frac{m^{2} \delta P}{r^{2}}=0
$$

Now, by taking the divergence of Eq. (15), and using Eqs. (10) and (34), one finds that, up to terms of second order in $\varepsilon$, one has $\mathbf{e}_{z} \cdot \nabla \times \xi_{\perp}=0$, i.e.,

$$
\xi_{\perp}=-\nabla_{\perp} \chi
$$

where $\chi$ is some scalar function. Taking the divergence of this equation, one finds that, up to terms of second order in $\varepsilon$,

$$
\nabla_{\perp}^{2} \chi \equiv \frac{1}{r} \frac{\partial}{\partial r} r \frac{\partial \chi}{\partial r}-\frac{m^{2} \chi}{r^{2}}=0
$$

Solution of this equation that satisfies the boundary condition (19), $\partial \chi /\left.\partial r\right|_{r=c}=0$, is:

$$
\chi=C_{2}(z)\left(r^{m}+\frac{c^{2 m}}{r^{m}}\right)
$$


The coefficient $\mathrm{C}_{2}$ can be expressed in terms of the radial displacement of the boundary, i.e., in terms of the function (31):

$$
C_{2}(z)=-\frac{\xi_{0}(z)}{m a^{m-1}\left(1-\varsigma^{m}\right)}
$$

where $\zeta$ is defined by Eq. (7). The perpendicular displacement $\boldsymbol{\xi}_{\perp}$, according to (35), is proportional to $\xi_{0}(z)$; therefore, the boundary condition $\left.\xi_{\perp}\right|_{z=0, L}=0$ will be satisfied for any radius $r$ just by setting $\xi_{0}(z=0, L)=0$. This is a feature of the long-thin approximation. By returning to Eq. (15), one then finds the following relationship between $\xi_{0}$ and $\delta P(a, z):$

$$
\delta P(a)=\frac{a}{m}\left[\left(\omega^{2} \rho+\frac{1}{4 \pi} \hat{Q}_{i}^{2}\right) \frac{1+\zeta^{m}}{1-\zeta^{m}}+i \frac{B_{\varphi i}}{2 \pi a} \hat{Q}_{i}\right] \xi_{0} .
$$

Finally, by using Eq. (32), one finds the following eigen-equation for $\xi_{0}$ :

$$
\left[\left(\omega^{2} \rho+\frac{1}{4 \pi} \hat{Q}_{i}^{2}\right) \frac{1+\zeta^{m}}{1-\zeta^{m}}+i \frac{B_{\varphi i}}{2 \pi a} \hat{Q}_{i}\right] \xi_{0}=\left(-\frac{1}{4 \pi} \frac{1+\eta^{m}}{1-\eta^{m}} \hat{Q}_{e}^{2}+m \frac{B_{\varphi i}^{2}-B_{\varphi e}^{2}}{4 \pi a^{2}}\right) \xi_{0}
$$

This equation is the basis for the stability analysis in a long-thin approximation.

In order not to introduce additional parameters of order 1 into the problem, we assume that

$$
B_{z e}=B_{z i}=B_{z}
$$

This assumption corresponds to a low-pressure plasma, typical of long-thin pinch equilibria. An analysis of the general case (in terms of the $B_{z e} / B_{z i}$ ratio) does not raise any significant new insights; it just makes all the calculations more lengthy. Also, the assumption (41) is the one normally made in reference to the "standard" KS criterion (Cf. Ref. [3]). Equation (40), with the condition (41) imposed, can then be rewritten as: 


$$
\frac{\partial^{2} \xi_{0}}{\partial z^{2}}-2 i K \frac{\partial \xi_{0}}{\partial z}+\left[\alpha_{2} \frac{4 \pi \rho \omega^{2}}{B_{z}^{2}}-\tilde{K}^{2}\right] \xi_{0}=0
$$

where

$$
\begin{aligned}
& K=\alpha_{1} \frac{B_{\varphi e}}{a B_{z}}\left[D\left(m \frac{1+\zeta^{m}}{1-\zeta^{m}}-1\right)+m \frac{1+\eta^{m}}{1-\eta^{m}}\right] \\
& \tilde{K}^{2}=\alpha_{1} m\left(\frac{B_{\varphi e}}{a B_{z}}\right)^{2}\left[D^{2}\left(m \frac{1+\zeta^{m}}{1-\zeta^{m}}-1\right)+\left(m \frac{1+\eta^{m}}{1-\eta^{m}}-1\right)\right] \\
& \alpha_{1}=\left(\frac{1+\varsigma^{m}}{1-\varsigma^{m}}+\frac{1+\eta^{m}}{1-\eta^{m}}\right)^{-1} ; \alpha_{2}=\frac{1+\varsigma^{m}}{1-\varsigma^{m}} \alpha_{1},
\end{aligned}
$$

and coefficients $D, \eta$, and $\zeta$ are defined by Eqs. (4), (7).

\section{THE CASE OF ZERO VOLUMETRIC CURRENT AND NO CENTRAL ROD}

This case deserves special attention for two reasons: first, this case is the one normally considered in the derivation of the Kruskal-Shafranov stability criterion (2) (See, e.g., Ref. 3). Second, calculations in this case are relatively brief and allow one to get to the essence of the problem without being trapped in lengthy equations. For the same reason, we also assume that the central rod is absent. In this case, one has $\zeta=0$ and $D=0$, so that

$$
K=\frac{m\left(1+\eta^{m}\right)}{2} \frac{B_{\varphi e}}{a B_{z}} ; \quad \tilde{K}^{2}=\left(\frac{B_{\varphi e}}{a B_{z}}\right)^{2} m\left(m-\frac{1-\eta^{m}}{1+\eta^{m}}\right) ; \quad \alpha_{1}=\alpha_{2}=\frac{1-\eta^{m}}{2}
$$

\section{A. Reproducing the Kruskal-Shafranov stability condition}

We first demonstrate that our approach reproduces the KS stability criterion. For the infinitely long pinch, one can seek a solution of Eq. (42) in the form $\exp (i k z)$. This yields the following dispersion relation: 


$$
k^{2}-2 k K+\tilde{K}^{2}-\alpha_{2} \frac{4 \pi \rho \omega^{2}}{B_{z}^{2}}=0
$$

Using Eq. (46) one finds that

$$
\omega^{2}\left(1-\eta^{m}\right)=\frac{B_{z}^{2}}{2 \pi \rho a^{2}}\left[k a-\frac{m}{2} \frac{B_{\varphi e}}{B_{z}}\left(1+\eta^{m}\right)\right]^{2}+m \frac{B_{\varphi e}^{2}}{2 \pi \rho a^{2}}\left[\frac{m\left(1+\eta^{m}\right)}{2}-1\right]\left(1-\eta^{m}\right) .
$$

For $m \geq 2$ the system is obviously stable (the RHS is manifestly non-negative). For $m=1$, Eq. (48) can be written as:

$$
\omega^{2}(1-\eta)=\frac{B_{z}^{2}}{2 \pi \rho a^{2}}\left\{k^{2} a^{2}-k a \frac{B_{\varphi e}}{B_{z}}(1+\eta)+\eta\left(\frac{B_{\varphi e}}{B_{z}}\right)^{2}\right\} .
$$

For any given $k$, at a small enough current ( $\operatorname{small} B_{\varphi e}$ ), the system is stable. However, with a growing current (growing $B_{\varphi e}$ ) the r.h.s. of Eq. (49) eventually becomes zero, corresponding to marginal stability. This happens at

$$
B_{\varphi e}=k a B_{z e}
$$

Note that the parameter $\eta$ has dropped out from this expression.

If one now imposes the periodicity condition (1), one finds from (50) the standard Kruskal-Shafranov expression (2) for the critical current (Cf. Eqs. (7.7)-(7.9) of Ref. [3]).

The dispersion relation (49) predicts stability if, for a given $k$, the current becomes larger than a certain level, so that $B_{\varphi e} / B_{z}>k a / \eta$. For $k=2 \pi / L$, the stability reappears at

$$
\frac{I}{I_{K S}}>\frac{1}{\eta}
$$

This happens because of the stabilizing effect of the outer conducting shell. When the shell is very far away, $\eta \rightarrow 0$, the stabilizing effect disappears. As we shall see in the next section, the reappearance of stability is entirely related to the use of periodic boundary conditions. 


\section{B. The effect of boundary conditions}

Now we return to Eq. (42) and recall that one has to impose two boundary conditions,

$$
\xi_{0}(z=0)=0 ; \xi_{0}(z=L)=0,
$$

not just the periodicity constraint. The solution of Eq. (42) satisfying the boundary condition (52) at $z=0$ is

$$
\xi_{0}=C\left[\exp \left(i k_{1} z\right)-\exp \left(i k_{2} z\right)\right]
$$

where $C$ is an arbitrary constant and $k_{1,2}$ are the two solutions of Eq (47),

$$
k_{1,2}=K \pm \sqrt{K^{2}-\tilde{K}^{2}+\frac{4 \pi \alpha_{2} \rho \omega^{2}}{B_{z}^{2}}}
$$

To satisfy the second of the boundary conditions (52) (at $z=L)$, one has to have

$$
k_{1}-k_{2}=\frac{2 \pi}{L} n
$$

with $n=1,2,3, \ldots$. It is clear from Eqs. (54), (55) that $k_{1}$ and $k_{2}$ can be expressed as:

$$
k_{1}^{(n)}=K+\frac{n \pi}{L} ; k_{2}^{(n)}=K-\frac{n \pi}{L}
$$

The eigenfunctions

$$
f^{(n)}=\frac{e^{i K z}}{\sqrt{2}}\left(e^{\frac{i n \pi z}{L}}-e^{-\frac{i n \pi z}{L}}\right)
$$

form an ortho-normal set. The mode with the lowest stability threshold is, obviously, the $n=1$ mode. For it

$$
\frac{4 \pi \alpha_{2} \rho \omega^{2}}{B_{z}^{2}}=\tilde{K}^{2}-K^{2}+\left(\frac{\pi}{L}\right)^{2}
$$

Equations (47) and (52)-(58) pertain actually to arbitrary values of $D$ and $\zeta$, not only to $D=\zeta=0$. 
Returning to the case where $D=\zeta=0$, one finds from Eq. (58) the following expression for $\omega^{2}$ :

$$
\omega^{2}=\left(\frac{B_{\varphi e}^{2}}{4 \pi \rho a^{2}}\right)\left[\frac{m^{2}}{2}\left(1+\eta^{m}\right)-m+\frac{1}{2\left(1-\eta^{m}\right)}\left(\frac{2 \pi a B_{z}}{L B_{\varphi e}}\right)^{2}\right]
$$

For $m \geq 2$ the system is universally stable. For $m=1$, the dispersion relation becomes

$$
\boldsymbol{\omega}^{2}=\frac{\pi B_{z}^{2}}{2(1-\boldsymbol{\eta}) \rho L^{2}}\left[1-(1-\boldsymbol{\eta})^{2}\left(\frac{L B_{\varphi e}}{2 \pi a B_{z}}\right)^{2}\right]
$$

At large-enough current the system becomes unstable. This obviously happens at

$$
I>I_{c r i t} \equiv \frac{I_{K S}}{1-\eta}
$$

One sees that there is a substantial difference between Eqs. (2) and (61) in that the system with the boundary conditions properly imposed becomes more stable and the critical current becomes dependent on the geometrical factor $\eta$.

Another important difference with the case of a periodic boundary condition (Section IV.A) is that the system remains unstable at a large current (there is no upper stability boundary defined by Eq. (51)). This is so because, in the case of periodic boundary conditions, the mode structure was determined once and for all by Eq. (1) and didn't depend on the plasma current. Conversely, in the case of two boundary conditions (52) properly taken into account, the mode structure does change with the current. Eqs. (46) and (56) show that

$$
k_{1}^{(1)}=\frac{\pi}{L}\left[(1+\eta) \frac{I}{I_{K S}}+1\right] ; \quad k_{2}^{(1)}=\frac{\pi}{L}\left[(1+\eta) \frac{I}{I_{K S}}-1\right]
$$

At the stability boundary, $I / I_{K S}=(1-\eta)^{-1}$, one has 


$$
k_{1}^{(1)}=\frac{2 \pi}{L} \frac{1}{1-\eta} ; \quad k_{2}^{(1)}=\frac{2 \pi}{L} \frac{\eta}{1-\eta}
$$

The mode structure for several pinch currents is shown in Fig. 2 (b,c).

Only in the limit of a far-away wall, $\eta \rightarrow 0$, is the KS result recovered. This is easy to understand: in the case of a far-away wall, translation of the pinch in the $x$ or $y$ directions does not change the magnetic field outside the column (in the frame attached to the column axis). Then, by superposing such a translation with the purely helical perturbation $\exp (i k z-i \varphi), k=2 \pi / L$, one can adjust the translation in such a way that both foot points of the column would remain in their initial positions (i.e., a zero displacement boundary condition would be satisfied at the end plates). This structure of the unstable perturbation at $\eta=0$ automatically comes out of Eq. (63), where $k_{2}$ indeed becomes zero, meaning a mode which is a pure translation.

\section{Corrections to the long-thin model}

There are two types of corrections (in the parameter $\varepsilon$ ) to the expressions for the critical currents obtained in the previous section. The first correction comes from the fact that we have ignored the terms $\partial^{2} \delta P / \partial z^{2}$ and $\partial^{2} \psi / \partial z^{2}$ when solving equations $\nabla^{2} \delta P=0$ and $\nabla^{2} \psi=0$ (see, e.g., Eq. (28)). Retaining these terms will, obviously, give rise to corrections of order $\varepsilon^{2}$ to the zero-order approximation.

The second correction is related to the fact that we have not yet used the condition of zero normal displacement $\xi_{z}$ at the end walls (the second of Eqs. (18)). As one can check, our approximate solution does not satisfy this condition. Whence, the solution inside the plasma has to be adjusted at a distance $\sim a$ from the end-walls to satisfy this boundary condition. Analogously, our solution does not exactly satisfy the condition of 
zero perturbation of the normal component of the magnetic field at the surface of endplates in the vacuum region $(a<r<b)$ : in the solution (29) $\partial C_{1} / \partial z \neq 0$ at $z=0, L$. The combination of these factors is the second source of correction to the eigenfunction and to the critical current.

The corresponding adjustment to the eigenfunction occurs in the zone which is at a distance $\sim a$ from the end-plate. The eigenfunction $\xi_{0}(z)$ here is small, $\sim a / L$ compared to its maximum near the midplane. Accordingly, the correction to the critical current should be $\sim a^{2} / L^{2} \sim \varepsilon^{2}$ i.e. of the same order as the first correction. Therefore, one can expect that our results for the critical current have an accuracy $\sim O\left(\varepsilon^{2}\right)$.

\section{The energy principle}

To consider the problem in a more quantitative fashion, one can use the energy principle. We pursue this approach for the case of a zero distributed current and derive the energy principle free of any assumptions regarding the aspect ratio. The potential energy of perturbations, $W$, is expressed as an one-dimensional functional, in terms of a single function, $\xi_{0}(z) \equiv \xi_{r}(r=a, z)$.

Our plan is to express the pressure perturbation in the plasma and the magnetic field perturbation in the vacuum in terms of the displacement of the plasma boundary, $\xi_{0}(z) \equiv \xi_{r}(z, a)$. We will then use the following expression for the energy perturbation:

$$
W=-\pi a \int_{0}^{L} d z\left\{\xi_{0}^{*}\left[\delta P-\frac{1}{4 \pi}\left(\mathbf{B}_{e} \cdot \delta \mathbf{B}_{e}-\frac{B_{\varphi e}^{2}}{a} \xi_{0}\right)\right]+c . c .\right\},
$$

where $\delta P$ and $\delta \boldsymbol{B}_{e}$ are taken at $r=a$. This is nothing more than the work performed by the virtual displacement of the interface against the imbalanced pressure force, Eq. (20). Equation (64) can be easily reduced to the standard expression for the potential energy 
perturbation as presented, e.g., in Refs. 3, 4. The system is unstable if $W$ is negative. The stability boundary corresponds to the case where the maximum of $W$ over all the trial functions is zero. As all the quantities in the RHS will be expressed in terms of the displacement of the boundary, $\xi_{0}(z)$, Eq. (64) is a one-dimensional functional. All the boundary conditions will be folded into this equation - whence the convenience of this representation.

For the case of zero distributed current $\left(B_{\varphi i}=0\right), \delta P$ satisfies the Laplace equation (as is clear from Eqs. (12) and (10)):

$$
\frac{1}{r} \frac{\partial}{\partial r} r \frac{\partial \delta P}{\partial r}-\frac{m^{2} \delta P}{r^{2}}+\frac{\partial^{2} \delta P}{\partial z^{2}}=0
$$

The boundary conditions for this equation in the radial coordinate are the absence of a singularity at $r=0$ and the condition

$$
\left.\frac{\partial P}{\partial r}\right|_{r=a}=\frac{B_{z}^{2}}{4 \pi} \frac{\partial^{2} \xi_{0}(z)}{\partial z^{2}}
$$

at $r=a$. The latter condition is just the radial component of Eq. (15) with $\omega=0$ and $B_{\varphi}=0$, taken at $r=a ; \xi_{0}$, as before, is $\xi_{r}(z, a)$.

To find the boundary conditions in the $z$ direction, we consider the $z$ component of Eq. (15), which reads:

$$
\frac{\partial P}{\partial z}=\frac{B_{z}^{2}}{4 \pi} \frac{\partial^{2} \xi_{z}}{\partial z^{2}}
$$

Integrating it once and taking into account the first of the boundary conditions (18) and Eq. (10), one finds that

$$
\delta P(r, z)=\delta P(r, 0)+\frac{B_{z}^{2}}{4 \pi} \frac{\partial \xi_{z}(r, z)}{\partial z}
$$


and $\delta P(r, 0)=\delta P(r, L)$. Integrating Eq. (68) and taking into account the second of the boundary conditions (18), one then finds two boundary conditions for $\delta P$ :

$$
\delta P(r, 0)=\delta P(r, L)=\frac{1}{L} \int_{0}^{L} \delta P(r, z) d z
$$

The two boundary conditions (69) and the two radial boundary conditions (Eq. (66) and regularity at $r=0$ ) allow us, in principle, to express the pressure perturbation in terms of the displacement of the boundary of the plasma column.

We decompose the perturbation of the effective pressure $\delta P(r, z)$, as a function of $r$, over a complete orthogonal set of functions $J_{m}\left(\mu_{s} r / a\right)$, where $J_{m}$ is the Bessel function and $\mu_{\mathrm{s}}$ is the $s$-th root of the equation $J_{m}^{\prime}\left(\mu_{s}\right)=0, s=1,2, \ldots$ As we shall see below, the use of this basis allows one to naturally fold into the analysis the boundary condition (66). To find the Fourier coefficients $F_{s}(z)$ in the expansion

$$
\delta P=\sum_{s} J_{m}\left(\mu_{s} r / a\right) F_{s}(z)
$$

one has to multiply Eq. (65) by $r J_{m}\left(\mu_{s} r / a\right)$ and integrate from $O$ to $a$. In this way, after two integrations by parts, and taking into account the boundary condition (66), one obtains

$$
\frac{\partial^{2} F_{s}}{\partial z^{2}}-\frac{\mu_{s}^{2}}{a^{2}} F_{s}=-\frac{u_{s} B_{z}^{2}}{4 \pi a} \frac{\partial^{2} \xi_{0}}{\partial z^{2}}
$$

where

$$
u_{s}=J_{m}\left(\mu_{s}\right)\left[\int_{0}^{1} x J_{m}^{2}\left(\mu_{s} x\right) d x\right]^{-1}
$$

For brevity, we skip the subscript " $m$ " on $\mu, F$, and other similar quantities. 
The lengthy solution of Eq. (71) subject to boundary conditions (69) imposed on the functions $F_{s}$ is presented in the Appendix.

In the vacuum region we decompose the potential $\psi$ over an orthogonal set of functions

$$
Y_{m} \equiv \frac{J_{m}\left(v_{s} r / b\right)}{J_{m}^{\prime}\left(v_{s}\right)}-\frac{N_{m}\left(v_{s} r / b\right)}{N_{m}^{\prime}\left(v_{s}\right)}
$$

where $v_{s}$ is the $s^{\prime}$ th root of the equation

$$
\frac{J_{m}^{\prime}\left(v_{s} a / b\right)}{J_{m}^{\prime}\left(v_{s}\right)}-\frac{N_{m}^{\prime}\left(v_{s} a / b\right)}{N_{m}^{\prime}\left(v_{s}\right)}=0
$$

The function $Y_{m}$ has a zero derivative at $r=a, b$. One has:

$$
\psi=\sum_{s} Y_{m}\left(v_{s} r / b\right) G_{s}(z)
$$

Multiplying Eq. (22) by $r Y_{s}$, integrating from $r=a$ to $r=b$, and taking into account the boundary conditions (23) and (24), we find that $G_{s}$ satisfies equation:

$$
\frac{\partial^{2} G_{s}}{\partial z^{2}}-\frac{v_{s}^{2}}{b^{2}} G_{s}=-\frac{w_{s}}{a}\left(B_{z} \frac{\partial \xi_{0}}{\partial z}-\frac{i m \xi_{0}}{a} B_{\varphi e}\right),
$$

where

$$
w_{s}=\eta Y_{m}\left(v_{s} \sqrt{\eta}\right)\left[\int_{\sqrt{\eta}}^{1} x Y_{m}^{2}\left(v_{s} x\right) d x\right]^{-1}
$$

The solution of Eq. (76) is presented in Appendix 1.

Inserting the functions (70) and (75) [which are now completely defined by virtue of Eqs.(A.1) and (A.3)] in Eq. (64), one finds a general expression for the functional (64), free of any assumptions regarding the parameter $\varepsilon$. We leave the analysis of the aspect ratio $\sim 1$ for the future work. Here we limit ourselves just to a comment that, in order to 
find the stability margin at the aspect ratio $\sim 1$, one can use the Galerkin-Ritz approach, with the basis functions (57).

\section{E. Evaluating terms $\sim \varepsilon^{2}$}

\section{Evaluating $F_{s}$ and $G_{s}$}

To evaluate the terms $\sim \varepsilon^{2}$, we substitute the solution (53), with $k_{1,2}$ as in Eq. (62), as a trial function into the functional (64). As the function (53) is not an exact solution, what we find is not the minimum value of the functional. Therefore, if we set our approximate expression for $W$ to zero, and find from this equation the azimuthal magnetic field $B_{\varphi e}$, the result will be an upper bound estimate for the critical field (and, accordingly, to the critical current).

As in a slender pinch without distributed current only the $m=1$ mode is unstable, in the rest of this section we assume that $m=1$. For $\xi_{0}$ as in Eq. (53), with $C=1$, the expressions for $F_{s}$ and $G_{s}$ [which determine the functions $\delta P$ and $\psi$ via Eqs. (70) and (75)] read:

$$
\begin{aligned}
& F_{s}=-\frac{u_{s} B_{z}^{2}}{4 \pi a \kappa_{s}^{2}}\left[k_{1}^{2} e^{i k_{1} z}-k_{2}^{2} e^{i k_{2} z}\right]+ \\
& \left\{A_{+} e^{\kappa_{s}(z-L)}+A_{-} e^{-\kappa_{s} z}+\frac{u_{s} B_{z}^{2}}{4 \pi a \kappa_{s}^{4}}\left(k_{1}^{4} e^{i k_{1} z}-k_{2}^{4} e^{i k_{2} z}\right)\right\} \\
& G_{s}=\frac{i w_{s}}{a q_{s}^{2}}\left[\left(k_{1} B_{z}-\frac{B_{\varphi e}}{a}\right) e^{i k_{1} z}-\left(k_{2} B_{z}-\frac{B_{\varphi e}}{a}\right) e^{i k_{2} z}\right]+ \\
& \left\{B_{+} e^{q_{s}(z-L)}+B_{-} e^{-q_{s} z}+\frac{i w_{s} k_{1}^{2}}{a q_{s}^{4}}\left(k_{1} B_{z}-\frac{B_{\varphi e}}{a}\right) e^{i k_{1} z}-\frac{i w_{s} k_{2}^{2}}{q_{s}^{4}}\left(k_{2} B_{z}-\frac{B_{\varphi e}}{a}\right) e^{i k_{2} z}\right\} \\
& A_{ \pm}=\frac{u_{s} B_{z}^{2}}{4 \pi a \kappa_{s}^{2}}\left(k_{1}^{2}-k_{2}^{2}\right) L e^{i k_{2} L \sigma_{ \pm}} ; \sigma_{+}=1 ; \sigma_{-}=0
\end{aligned}
$$




$$
B_{ \pm}= \pm \frac{w_{s} e^{i k_{2} L \sigma_{ \pm}}}{a q_{s}^{3}}\left[\left(k_{1}^{2}-k_{2}^{2}\right) B_{z}-\left(k_{1}-k_{2}\right) \frac{B_{\varphi e}}{a}\right] ; \quad \sigma_{+}=1 ; \sigma_{-}=0
$$

where $u_{s}$ and $w_{s}$ are determined by Eqs. (72) and (77), and $\kappa_{s}=\mu_{s} / a, q_{s}=v_{s} / b$. For brevity, we skip the superscript "(1)" on $k_{l, 2}$.

We have used several approximations in the derivation of these expressions:

i) As we are interested in large values of the parameters $\kappa_{s} L, q_{s} L$, we have neglected terms that are exponentially small in these parameters. ii) The terms containing coefficients $A_{ \pm}, B_{ \pm}$are substantially different from zero only near the walls, at distances $\sim \varepsilon L$. At these distances, the function $\xi_{0}$ is of order of $\varepsilon$. Therefore, the contribution of these terms to the energy functional is of order of $\varepsilon^{2}$, in agreement with the qualitative discussion at the beginning of this section. Since we are looking for corrections of order of $\varepsilon^{2}$, we have neglected the higher-order (in $\varepsilon$ ) corrections to the coefficients $A_{ \pm}, B_{ \pm}$. iii) In other terms, we have retained only contributions up to the order $\varepsilon^{2}$.

\section{Recovering the results of a long-thin approximation}

We arranged the RHS of Eqs. (78), (79) in such a way that the terms assembled in the square brackets are the leading order terms and should yield the stability condition of the long-thin approximation, whereas terms assembled in the curly brackets are responsible for the correction of order $\varepsilon^{2}$.

The first group of terms, when substituted to the series (70), (75) yields the following expression for $\delta P(a)$ and $\psi$ :

$$
\begin{aligned}
& \delta P(a, z)=-\frac{a B_{z}^{2}}{4 \pi}\left(k_{1}^{2} e^{i k_{1} z}-k_{2}^{2} e^{i k_{2} z}\right) \sum_{s} \frac{J_{1}\left(\mu_{s}\right) u_{s}}{\mu_{s}^{2}} \\
& \psi(a, z)=\frac{i b^{2}}{a}\left[\left(k_{1} B_{z}-\frac{B_{\varphi e}}{a}\right) e^{i k_{1} z}-\left(k_{2} B_{z}-\frac{B_{\varphi e}}{a}\right) e^{i k_{2} z}\right] \sum_{s} \frac{Y_{1}\left(v_{s} \sqrt{\eta}\right) W_{s}}{v_{s}^{2}}
\end{aligned}
$$


Using the identities (A.6) and (A.9), one finds that

$$
\begin{aligned}
& \delta P(z, a)=-a \frac{B_{z}^{2}}{4 \pi}\left(k_{1}^{2} e^{i k_{1} z}-k_{2}^{2} e^{i k_{2} z}\right), \\
& \psi(z, a)=i a \frac{1+\eta}{1-\eta}\left[\left(k_{1} B_{z}-\frac{1}{a} B_{\varphi e}\right) e^{i k_{1} z}-\left(k_{2} B_{z}-\frac{1}{a} B_{\varphi e}\right) e^{i k_{2} z}\right]
\end{aligned}
$$

This allows one to find the quantity $\delta P-\frac{1}{4 \pi}\left(\mathbf{B}_{e} \cdot \delta \mathbf{B}_{e}-\frac{B_{\varphi e}^{2}}{a} \xi_{0}\right)$ in the integrand of (64) and to perform an integration over $z$ [we recall that $\xi_{0}$ is determined by Eq. (53), with $k_{1,2}$ determined by Eq. (62)]. Skipping simple but lengthy elementary integrations (when making them, one extensively uses condition $\left.k_{1}-k_{2}=2 \pi / L\right)$, one finds the following expression for the potential energy:

$$
\begin{aligned}
& W^{(0)}=\frac{2 \pi a^{2} L}{1-\eta} \frac{B_{z}^{2}}{4 \pi}\left[\left(k_{1}^{2}+k_{2}^{2}\right)-(1+\eta)\left(k_{1}+k_{2}\right) \frac{B_{\varphi e}}{a B_{z}}+2 \eta\left(\frac{B_{\varphi e}}{a B_{z}}\right)^{2}\right]= \\
& \frac{4 \pi^{3} a^{2}}{L(1-\eta)} \frac{B_{z}^{2}}{4 \pi}\left[1-(1-\eta)^{2}\left(\frac{I}{I_{K S}}\right)^{2}\right]
\end{aligned}
$$

At the last step, we have expressed the azimuthal magnetic field in terms of the axial current and explicitly used Eq. (62).

At a low plasma current, the potential energy (87) is, obviously, positive. It turns zero when the plasma current is exactly equal to that of Eq. (61). This is a test of consistency of the two approaches used in this paper: the long-thin approximation, and an energy principle approach, where the same result is obtained from the general expression for the potential energy. 


\section{Corrections $\sim \varepsilon^{2}$}

Now we switch to the onerous task of evaluating the correction $\sim \varepsilon^{2}$. We first substitute the terms in the curly brackets in Eqs. (78) and (79) into Eqs. (70) and (75), respectively, and obtain the second-order corrections to $\delta P$ and $\psi$. Then we substitute these second order terms into Eq. (64) and perform an integration. The result is the second-order correction to $W^{(2)}$ :

$$
\begin{aligned}
& W^{(2)}=-2 \pi a L\left[k_{1}^{4}+k_{2}^{4}-\frac{2 \pi}{L^{2}}\left(k_{1}^{2}-k_{2}^{2}\right) \sin 2 k_{2} L\right]\left[\sum_{s} \frac{u_{s} J_{1}\left(\mu_{s}\right)}{\kappa_{s}^{4}}\right]-2 \pi a L \frac{1}{4 \pi} \times \\
& \left\{k_{1}^{2}\left(k_{1} B_{z}-\frac{B_{\varphi e}}{a}\right)^{2}+k_{2}^{2}\left(k_{2} B_{z}-\frac{B_{\varphi e}}{a}\right)^{2}+\frac{2 \pi B_{z}}{L^{2}}\left[k_{1}\left(k_{1} B_{z}-\frac{B_{\varphi e}}{a}\right)+k_{2}\left(k_{2} B_{z}-\frac{B_{\varphi e}}{a}\right)\right] \cos 2 k_{2} L\right\} \times \\
& \left(\sum_{s} \frac{w_{s} Y_{1}\left(v_{s} \sqrt{\eta}\right)}{q_{s}^{4}}\right)
\end{aligned}
$$

As this is a second-order correction, we can plug into it the zeroth-order expression of the critical current, i.e., set $B_{\varphi e}=[2 \pi a / L(1-\eta)] B_{z e}$ and substite $k_{1,2}$ from Eq. (62). Note that the Bessel function series in Eq. (88) are rapidly converging, because they are alternating and, in addition, their members scale as $1 / s^{4}$. Finally, from the equation $\delta I^{(2)}\left(\partial W^{(0)} / \partial I\right)=-W^{(2)}$, one finds the second-order correction to the current.

We will not present here a very lengthy explicit expression for the correction. We note that one cannot use this analysis at $\eta$ too close to 1 (in other words, at too small a gap $b$ - $a$ between the plasma surface and the return current conductor). This is because we are limited to an approximation $k_{1,2} a<<1$, whereas at $1-\eta<<1$ the wave numbers $k_{1,2}$, according to Eq. (63), scale as $(2 \pi / L)(1-\eta)^{-1}$. Therefore, the applicability condition for Eq. (88) is: 


$$
1-\eta>\frac{2 \pi a}{L}
$$

\section{THE LONG-THIN APPROXIMATION AT $D \neq 0, \zeta \neq 0$}

In this section we return to the long-thin approximation but for arbitrary $D$ (Eq. (4)) and $\zeta$ (Eq. (7)). The corresponding stability criterion can be obtained from Eqs. (58) and (43)-(45). For the case where there is no central rod $(\zeta=0)$ these equations yield:

$$
\omega^{2}=\frac{B_{z}^{2}}{4 \pi \rho}\left(\frac{\pi}{L}\right)^{2} \frac{2}{1-\eta^{m}}\left[1-R\left(\frac{I}{I_{K S}}\right)^{2}\right]
$$

The parameter $R$ is:

$$
R=D^{2}\left(1-\eta^{m}\right)^{2}+2 m\left(1-\eta^{m}\right)(1-D)\left(1-\eta^{m} D\right)-m^{2}(1-D)^{2}\left(1-\eta^{2 m}\right)
$$

The stability boundary is determined by the equation

$$
\frac{I}{I_{K S}}=\frac{1}{\sqrt{R}}
$$

The boundary depends on the current distribution. For the hollow distribution $(D=0)$ only the $m=1$ mode is unstable; the instability criterion coincides with Eq. (61). For the uniform current $(D=1)$ all non-zero $m$ 's become unstable simultaneously, at the current determined by Eq. (2). The general case is illustrated by Fig. 4.

Consider now the situation where the central rod is present $(\zeta \neq 0)$. We start from the hollow distribution $(D=0)$. In this case Eq. (58) yields:

$$
\left(\frac{I}{I_{K S}}\right)^{2}=\frac{\left(1-\eta^{m} \zeta^{m}\right)^{2}}{m\left(1-\eta^{m}\right)\left(1-\zeta^{m}\right)\left[2\left(1-\eta^{m} \zeta^{m}\right)-m\left(1+\eta^{m}\right)\left(1+\zeta^{m}\right)\right]}
$$

Clearly, only the $m=1$ mode can be unstable. The critical current for it can be found from the following expression: 


$$
\frac{I}{I_{K S}}=\frac{1-\eta \zeta}{\sqrt{(1-\eta)(1-\zeta)(1-\eta-\zeta-3 \eta \zeta)}}
$$

A significant stabilizing effect requires quite a thick rod. For example, to increase the critical current by a factor of 2 over the Kruskal-Shafranov limit at $\eta=0$, requires $\zeta=0.75$, i.e., a rod with the radius $c=0.87 a$.

An interesting fact is that the system is formally stable at an arbitrarily high axial current (we call this "a robust stability") if both $\eta$ and $\zeta$ are large enough, so that

$$
\eta+\zeta+3 \eta \zeta>1
$$

The current in this domain can exceed the Kruskal-Shafranov current by a large factor. Still, in order for our analysis to be valid the condition $B_{\varphi e}<<B_{z}$ stemming from our ordering must be satisfied.

The condition of robust stability for an arbitrary value of the parameter $D$ can be easily obtained from Eq. (58) and reads as:

$$
m^{2} \frac{1+\varsigma^{m}}{1-\varsigma^{m}} \frac{1+\eta^{m}}{1-\eta^{m}}(D-1)^{2}-m(1-D)\left[\frac{1+\varsigma^{m}}{1-\varsigma^{m}}(1+D)+\frac{1+\eta^{m}}{1-\eta^{m}}(1-D)\right]-D^{2}>0
$$

Some of the numerical results related to the $m=1$ mode are shown on Fig. 5. We remind that the quantities $D$ and $\eta, \zeta$ are defined by Eqs. (4) and (7), respectively.

\section{DISCUSSION}

We have considered the ideal MHD stability of a pinch limited by two conducting end walls. It turned out that the presence of these walls has a strong effect on the stability limit and the mode structure even in a long-thin approximation. The reason is that the periodic boundary conditions that are usually imposed to mimic the finite length of the plasma system do not allow for zero plasma displacement at both end plates. 
In a long-thin case, there is a simple approach which can be used to eliminate this deficiency: As the $z$-dependence is weak (by the very nature of the long-thin limit) the perturbations can be locally (in $z$ ) considered as though there were no dependence on $z$ at all, and then the $z$-dependence would be re-introduced as a parameter. This approach yields an eigen-equation for the radial displacement of the column surface, $\xi_{0}(z)$; when the boundary conditions of the zero displacement at the end plates are imposed, one obtains a dispersion relation, which yields the critical current.

To extend this analysis to arbitrary aspect ratio, one can use the energy principle. We use it in the form where the potential energy perturbation is expressed in terms of the work performed by the radial displacement of the plasma-vacuum interface and obtain a one-dimensional functional expressed solely in terms of the function $\xi_{0}(z)$. This energy functional allows one to re-derive the expression for the critical current in the long-thin approximation, and estimate the accuracy of this approximation. The correction is of order of $(a / L)^{2}$ and depends on the geometrical parameter $\eta$.

Although our prime objective was a treatment of a formal theory problem, our results may be relevant to several experiments. In particular, in some regimes of spheromak discharges, the reconnection activity is associated with unstable $m=1$ helical perturbations of the central part of the discharge [9] where the field lines directly connect the top and the bottom of the flux conserver thereby mimicking the geometry of the screw pinch. To control this instability, one may try to use a conducting insert, which makes the central current-carrying column look like a hard-core pinch (Fig. 1b). However, experiments carried out at the SPHEX spheromak [10] have not found any strong effect of the central post. This may be not surprising in light of our results: 
according to Sec. V, although the presence of the core improves stability, in order this effect to become substantial (say, to increase the critical current by a factor of 2), the radius $c$ of the hard core has to be not far from the central column radius $a$, whereas in the SPHEX experiment it was more like $0.3 a$.

According to our results, a more efficient way of influencing the plasma stability would be to introduce a conducting stem with the radius equal to the radius of the central column (Fig. 6). If its height is, say, $L / 2$, one can expect a two-fold increase of the critical current. Such a techique could, in principle, be tried in the SSPX device [9].

Another experimental system for which our results may be relevant, is a University of Maryland experiment on the stabilization of Z-pinch instabilities by resistive moving walls [11]. Here our perfect conductivity analysis creates a reference point with respect to which finite resistivity effects could be studied.

\section{Acknowledgments}

The authors acknowledge helpful discussions with E.B. Hooper, L. LoDestro, M. Umansky, and S. Woodruff. We are also grateful to C. Sovinec for pointing out at the importance of end effects in the stability of the spheromak central column, and to C. Forest for drawing our attention to the University of Maryland experiment on the resistive wall effects in the pinch stability. This work was performed at UC LLNL for US DoE under contract W-7405-Eng-48. 


\section{APPENDIX}

The solution of Eq. (71) with the boundary conditions (69) and the function $\xi_{0}(z)$ satisfying the boundary condition $\xi_{0}(0)=\xi_{0}(L)=0$ is:

$$
F_{s}=\frac{U_{s}}{\kappa_{s}} \frac{C\left(e^{\kappa_{s} z}+e^{-\kappa_{s} z}\right)}{\sinh \kappa_{s} L+4 \frac{1-\cosh \kappa_{s} L}{\kappa_{s} L}}-\frac{U_{s}}{\kappa_{s}} \int_{0}^{z} \sinh \kappa_{s}\left(z-z^{\prime}\right) \frac{\partial^{2} \xi_{0}}{\partial z^{\prime 2}} d z^{\prime},
$$

where $\kappa_{s}=\mu_{s} / a$, and

$$
\begin{aligned}
& C=\frac{1-e^{-\kappa_{s} L}}{\kappa_{s} L} \int_{0}^{L}\left[\cosh \kappa_{s}\left(L-z^{\prime}\right)+\sinh \kappa_{s}\left(L-z^{\prime}\right)-1\right] \frac{\partial^{2} \xi_{0}}{\partial z^{\prime 2}} d z^{\prime}- \\
& \int_{0}^{L} \sinh \kappa_{s}\left(L-z^{\prime}\right) \frac{\partial^{2} \xi_{0}}{\partial z^{\prime 2}} d z^{\prime}
\end{aligned}
$$

The solution of Eq. (71) satisfying the boundary conditions (25) is:

$$
\begin{aligned}
& G_{s}=\frac{W_{s}}{q_{s}} \frac{\cosh q_{s} z}{\sinh q_{s} L} \int_{0}^{L}\left(B_{z} \frac{\partial \xi_{0}}{\partial z^{\prime}}-\frac{i m \xi_{0}\left(z^{\prime}\right)}{a} B_{\varphi e}\right) \cosh q_{s}\left(L-z^{\prime}\right) d z^{\prime}+ \\
& \frac{W_{s}}{q_{s}} \int_{0}^{z}\left(B_{z} \frac{\partial \xi_{0}}{\partial z^{\prime}}-\frac{i m \xi_{0}\left(z^{\prime}\right)}{a} B_{\varphi e}\right) \cosh q_{s}\left(z-z^{\prime}\right) d z^{\prime}
\end{aligned}
$$

where $q_{s}=v_{s} / b$.

To find the series of the Bessel functions that enter Eq. (83), we use the following approach. We note that the function $y(x)=x^{m}$ satisfies equation $\left(x y^{\prime}\right)^{\prime}-m^{2} / x=0$, where the prome means the differentiation over $x$. Multiplying this equation by $J_{m}\left(\mu_{s} x\right)$ and integrating over $x$ from 0 to 1, one finds that

$$
\mu_{s}^{2} \int_{0}^{1} x^{m+1} J_{m}\left(\mu_{s} x\right) d x=J_{m}\left(\mu_{s}\right)
$$

The second step is to decompose the function $y(x)=x^{m}$ over the functions $J_{m}\left(\mu_{s} x\right)$ on the segment $0 \leq x \leq 1$. Making this decomposition and setting $x=1$ in the final result, one obtains: 


$$
\sum_{s} J_{m}\left(\mu_{s}\right)\left[\int_{0}^{1} x^{m+1} J_{m}\left(\mu_{s} x\right) d x\right]\left[\int_{0}^{1} x J_{m}^{2}\left(\mu_{s} x\right) d x\right]^{-1}=1
$$

Substituting Eq. (A.4) into Eq. (A.5), one finds that

$$
\sum_{s} \frac{J_{m}\left(\mu_{s}\right) u_{s}}{\mu_{s}^{2}}=1
$$

To evaluate the series that enters Eq. (84), one applies the same technique to the function $y(x)=x^{m}+x^{-m}$, on the segment $\sqrt{\eta} \leq x \leq 1$. The results are:

$$
\begin{gathered}
\int_{1}^{\sqrt{1 / \eta}} x\left(x^{m}+\frac{1}{x^{m}}\right) Y_{m}\left(v_{s} x\right) d x=Y_{m}\left(v_{s} \sqrt{\eta}\right) \frac{m \sqrt{\eta}\left(1-\eta^{m}\right)}{v_{s}^{2}} \\
\sum_{s} Y_{m}\left(v_{s} \sqrt{\eta}\right)\left[\int_{\sqrt{\eta}}^{1} x\left(x^{m}+\frac{1}{x^{m}}\right) Y_{m}\left(v_{s} x\right) d x\right]\left[\int_{\sqrt{\eta}}^{1} x Y_{m}^{2}\left(v_{s} x\right) d x\right]^{-1}=\eta^{m / 2}+\frac{1}{\eta^{m / 2}} \\
\sum_{s} \frac{Y_{m}\left(v_{s} \sqrt{\eta}\right) w_{s}}{v_{s}^{2}}=\frac{\sqrt{\eta^{1-m}}\left(1+\eta^{m}\right)}{m\left(1-\eta^{m}\right)}
\end{gathered}
$$




\section{Figure captions}

Fig. 1 The geometry of the problem. a) A vertical cross-section of a pinch without a hard core. The shaded area represent perfectly conducting end plates (the top and the bottom) and a perfectly conducting shell. In the unperturbed state, there is a uniform vertical magnetic field frozen into the end electrodes. b) The geometry of a hard-core pinch. The shaded central column is perfectly conducting and may carry an axial current.

Fig. 2. Axial eigenfunctions at various currents. The real part is shown by the solid line, the imaginary part by the dashed line. a) An eigenfunction $\exp (2 \pi i z / L)$, i.e., an eigenfunction for periodic boundary conditions. Note that its real part is non-zero at $z=0, L$, meaning that this eigenfunction cannot be applied to the system with conducting end-plates. b) An eigenfunction (53) with $C=1, \eta=0.5$, and $I / I_{c r i t}=1$. c) An eigenfunction (53) with $C=1, \eta=0.5$ and $I / I_{\text {crit }}=2$.

Fig. 3. The axial current distributions analyzed in this paper. The current density is uniform in the pinch interior. There is a skin current flowing along the plasma surface. The parameter $D$ [Eq.(4)] is the ratio of the current in the interior to the total current (interior plus skin) and assumes the values between 0 and 1 .

Fig. 4. The stability boundary for various $D$ 's and $\eta$ 's. The sequence in which the modes become unstable at the growing current depends on the current distribution and the geometrical parameter $\eta$; in the case $\eta=0$, and $m=1$ one has $I_{c r i} / I_{K S}=1$, independently of $D$.

Fig. 5 The boundary of robust stability in the $(\eta, \zeta)$ plane for various current distributions for $m=1$ mode. The stable region is above the corresponding curve.

Fig. 6. A pinch with a partial central insert. Lightly shaded is the plasma column. 


\section{References}

1. M.D. Kruskal, M. Schwarzschild. Proc. Roy. Soc. London, A223, 348 (1954).

2. V.D. Shafranov, At. Energy, 5, 38 (1956).

3. B.B.Kadomtsev. In: Reviews of Plasma Phys. (M.A.Leontovich, Ed.), v.2,p.153, Consult. Bureau, NY, 1966.

4. J.P. Freidberg, Ideal Magnetohydrodynamics, NY, Plenum 1987

5. J.P. Freidberg, J.P. Goedbloed, R. Rohatgi. Phys. Rev. Lett., 51, 2105 (1983)

6. A.V. Arzhannikov et al, Trans. Fusion Techn., 35, 223, 1999

7. J.P. Freidberg, L.D. Pearlstein. Physics of Fluids 21, 1207 (1978)

8. I.B. Bernstein, E.A. Frieman, M.D. Kruskal, and R.M. Kulsrud. Proc. R. Soc. London, A244, 17 (1958)

9. D.N. Hill, R.H. Bulmer, B.I. Cohen, et al. "Spheromak Formation Studies in SSPX", in Proceedings of the 18th IAEA Fusion Energy Conference (Sorrento, 2000), paper ICP/09.

10. K.J. Gibson, P.K. Browning, S.J. Gee, S. Woodruff, D.M. Willett. Plasma Phys. Contr. Fus., 42, 1331 (2000).

11. W. Bergerson, C.B. Forest, D. Hannum, R.D. Hendrick, T.W. Lowell, S.P. Oliva, J.S. Sarff. Bull. Am. Phys. Soc., 48, 102 (2003) 

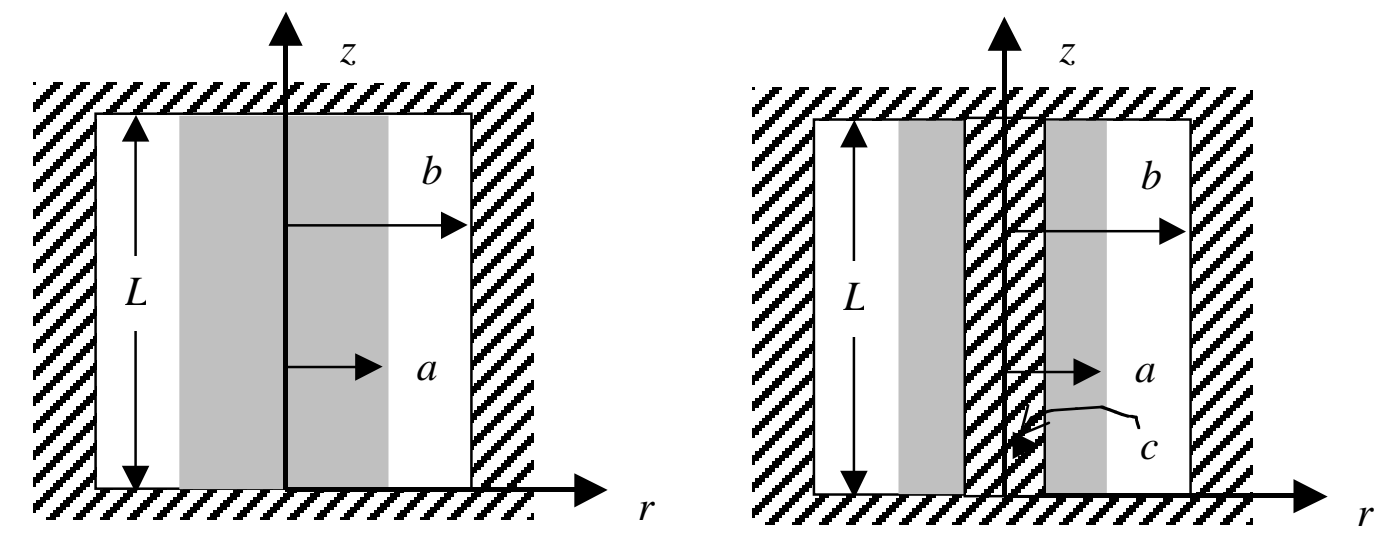

Fig. 1
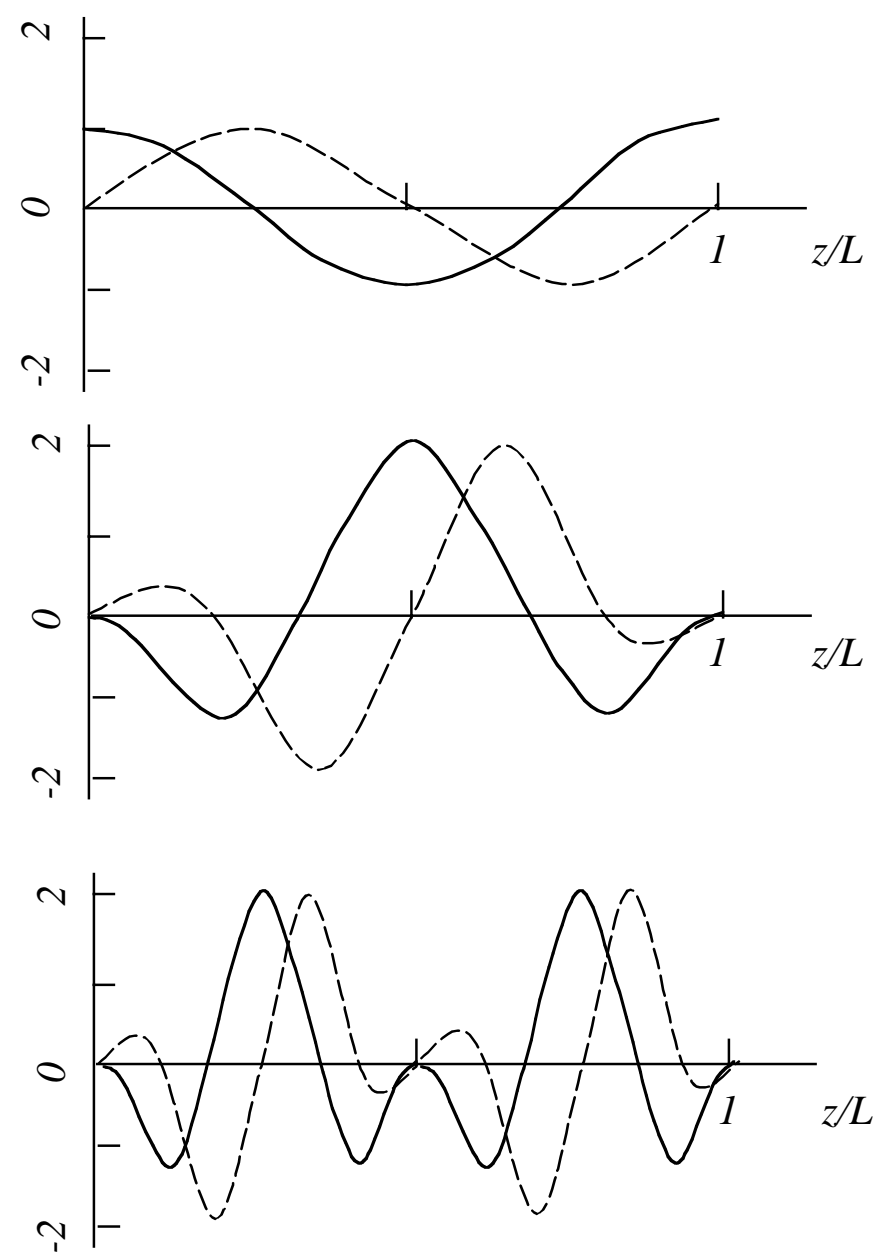

Fig. 2 


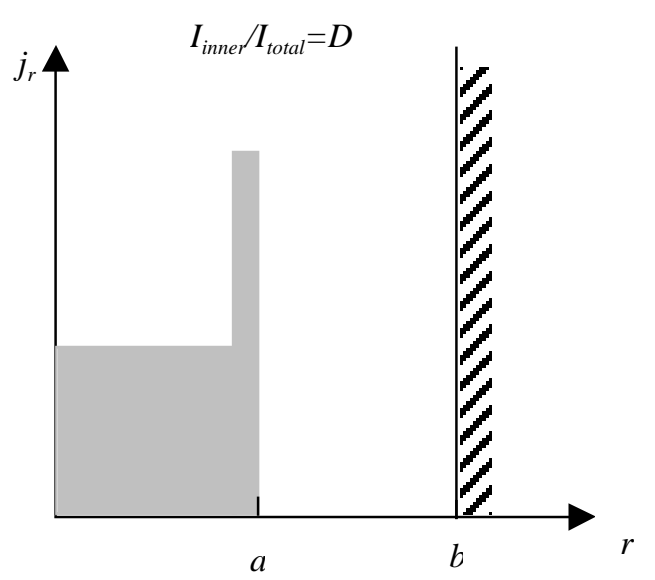

Fig. 3 

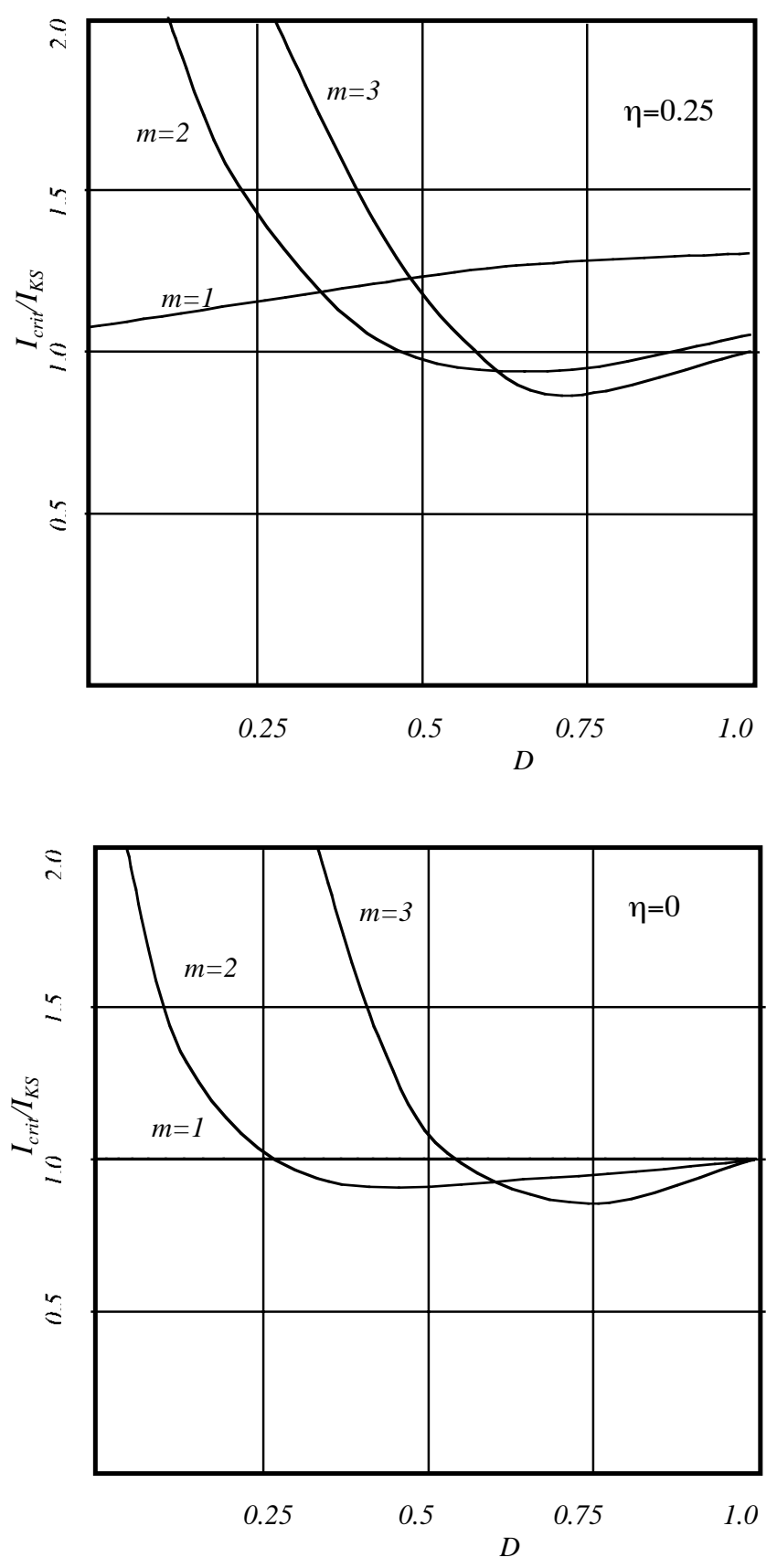

Fig. 4 


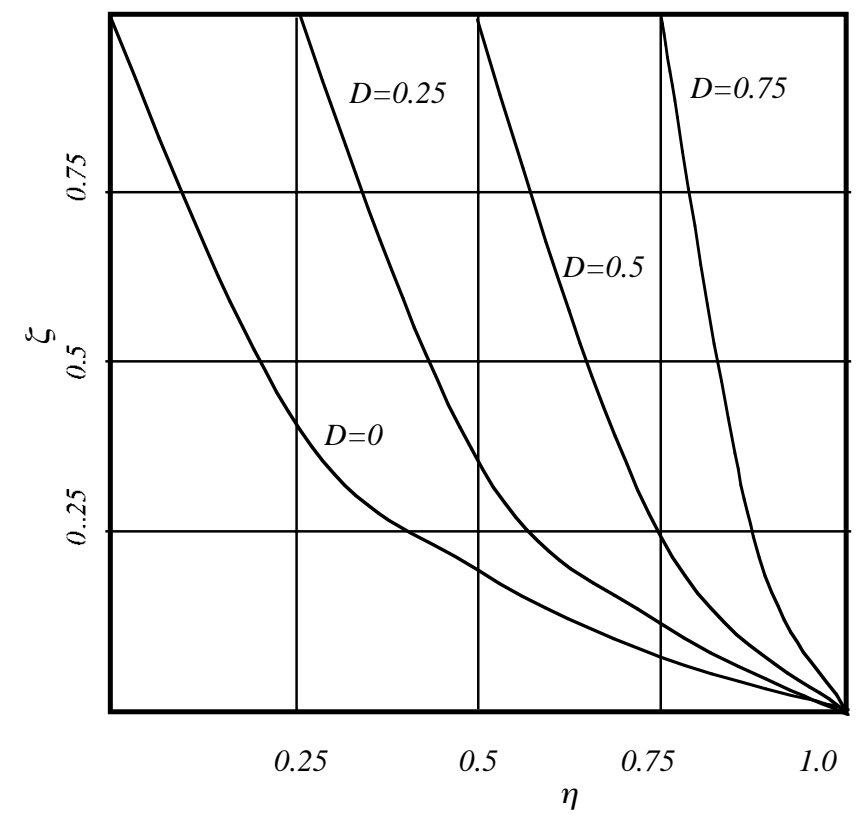

Fig. 5 


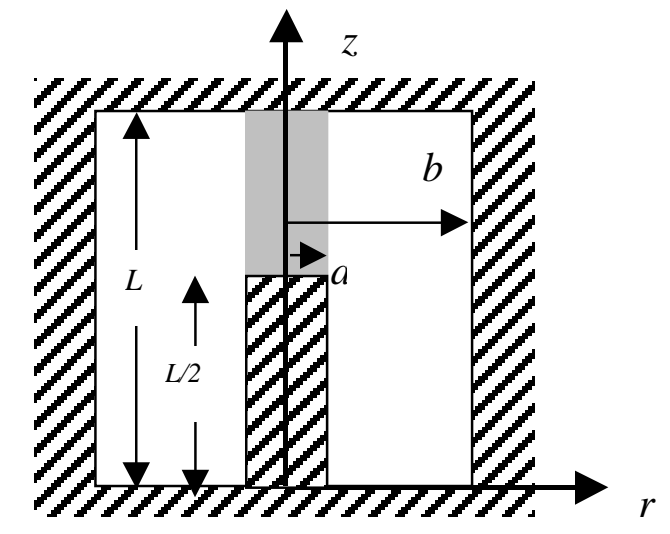

Fig. 6 